\title{
Antifreeze design for Muon Detector of LHAASO
}

\author{
Shaohui Feng ${ }^{* \dagger}$ \\ IHEP, CAS,(Institute of High Energy Physics, Chinese Academy of Sciences), for the LHAASO \\ Collaboration \\ E-mail: fengshdihep.ac.cn
}

Gang Xiao

IHEP, CAS

E-mail: 区iaogdihep.ac.cn

\section{Cong Li}

IHEP, CAS

E-mail: पicongdihep.ac.cn

\section{Xiong Zuo}

IHEP, CAS

E-mail: zuoxiongdihep.ac.cn

\section{Lingyu Wang}

IHEP, CAS

E-mail: wanglydihep.ac.cn

\section{Ning Cheng}

IHEP, CAS

E-mail: chengningdihep.ac.cn

\section{Huihai He}

IHEP, CAS

E-mail: hhhdihep.ac.cn

\section{Yi Zhang}

IHEP, CAS

E-mail: zhangyidihep.ac.cn

In the Large High Altitude Air Shower Observatory project, Muon Detectors will be built at $4400 \mathrm{~m}$ above sea level. As Water Cerenkov Detector, 44 tons of ultrapure water should be infused into the Muon Detector. In this cold area, once the water inside the Muon Detector freezes, consequence would be very serious. In this paper, a simulation of water temperature is built, the simulation data are consistent with the temperature data of Muon Detector at Yangbajing Tibet. Using the same simulation with air temperature, soil moisture and temperature of Dao Cheng, an antifreeze solution was designed. Through two years of testing, the design is successful.

35th International Cosmic Ray Conference - ICRC2017

10-20 July, 2017

Bexco, Busan, Korea

\footnotetext{
* Speaker.

$\dagger$ The LHAASO Collaboration
} 


\section{Introduction}

The Large High Altitude Air Shower Observatory (LHAASO) will explore basic problems such as the origin of high-energy cosmic rays and relevant evolution of the universe, with the highest sensitivity to ultra-high energy gamma rays, the highest survey sensitivity to very highenergy gamma ray sources, and wide cosmic ray energy coverage [四][] [B]. Covering a total area of $1.2 \mathrm{~km}^{2}$, LHAASO consists of a Kilometer-square Array (KM2A), a water Cherenkov Detector Array (WCDA) and a Wide-field Cherenkov telescope Array (WFCTA).

1,171 MDs will be built in the area with a spacing of $30 \mathrm{~m}$ throughout the whole array [四]. Such a large area of MDs would contribute to background-free observation of gamma rays, because gamma ray-induced showers contain scarce muons, whereas hadronic showers are abundant in muons [[]]. The number of muons in hadronic showers is correlated with the nucleon number of primary hadrons and can be used to discriminate the compositions of primary hadrons $[\mathrm{G}][\mathrm{W}]$, thus KM2A could help solve the problem in the cosmic rays spectrum at around the "knee" energy region $\left(10^{13}-10^{18} \mathrm{eV}\right)$. Furthermore, information on muon content in hadronic showers helps in

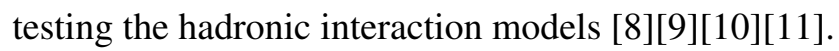

The MD adopts water Cherenkov technique to detect secondary muons in showers of cosmic rays. In order to achieve the main physical purposes, the MD unit should match the design as follows: Each muon detector includes a 6.8m-diameter concrete tank containing a sealed liner with a reflective inner surface, and the liner contains pure water. Cherekov light produced by the passage of particles through the water is collected by one eightinch-diameter PMT which is at the top center of the tank and looks downwards into the water through a window of optical EVA plastic. A $2.5 \mathrm{~m}$ thick shielding soil covers the tank to eliminate the electromagnetic components in showers.

Based on the design of MD, 44 tons of ultrapure water should be infused into the Muon detector. In this cold area, once the water inside the Muon detector freezes, consequence would be very serious.

In this paper, a simulation of water temperature is built, the simulation data are consistent with the temperature data of Muon detector at Yangbajing Tibet. Using the same simulation with air temperature, soil moisture and temperature of Dao Cheng, an antifreeze solution was designed. Through two years of testing, the design is successful.

\section{Thickness calculation for Antifreeze layer}

Conduction, convection and radiation are the three modes of heat transfer. Muon Detectors are buried in the deep ground, conduction is the main heat transfer pathway.

The Thermodynamics Empirical formula as follows:

$$
\begin{gathered}
Q=\frac{T_{1}-T_{w}}{\frac{1}{a_{1}}+\sum R+\frac{1}{a_{2}}} \\
\sum R=\left(d_{1}-\lambda_{1}\right)+\left(d_{1}-\lambda_{2}\right)+\cdots\left(d_{n}-\lambda_{n}\right)
\end{gathered}
$$

Where $Q$ is thermal flux, it means that when there is a temperature difference on both sides of the object, it is transmitted by heat through the object within the unit time. According to the 
antifreeze design specification, $Q=11 \mathrm{~W} / \mathrm{m}^{2} ; T_{1}$ is the lowest air temperature on the surface of the soil in winter. According to measurement data, $T_{1}=-27.6^{\circ} \mathrm{C}$, which is the lowest average temperature; $T_{w}$ is the lowest water temperature for MD in winter. Once the water inside the Muon Detector freezes, consequence would be very serious, $T_{w}$ should be $\geq 0^{\circ} \mathrm{C}$. In this paper, $T_{w}=$ $0^{\circ} \mathrm{C} ; a_{1}$ is Heat transfer coefficient between soil and air, $a_{1}=23.26 \mathrm{~W} / \mathrm{m}^{2} \cdot{ }^{\circ} C ; a_{2}$ is Heat transfer coefficient between soil and water, $a_{2}=58.15 \mathrm{~W} / \mathrm{m}^{2} \cdot{ }^{\circ} \mathrm{C} ; d_{n}$ are the thickness of different material; $\lambda_{n}$ are the thickness of different material;

Consider the Antifreeze performance and price, Polyurethane is used as an anti-freeze material for MD. As the Thermal conductivity is $0.04 \mathrm{~W} / \mathrm{m} \cdot \mathrm{K}$, Polyurethane, which is $100 \mathrm{~mm}$, is adopted to ensure that water is not frozen.

\section{Water Temperature simulation}

\subsection{Finite element simulation}

According to the MD design parameters, a transient thermal analysis model has been established.

Muon Detectors are buried in the ground, the temperature field is very complicated. In this paper, the temperature field is simplified:

a) The soil is a complex mixture, it belongs to the heterogeneous medium, in order to ease of calculation, the soil around MD is reduced to isotropic homogeneous medium;

b) Because of the symmetry on the both sides of MD, half of the vertical axis of the MD tank had been taken as a calculation area;

c) Assume that the MD tank is not moved after been set, and water transfer in soil is not been considered;

d) Assume that the temperature of MD water only affects the surrounding soil temperature, there is no affects when the distance is more than $15 \mathrm{~m}$;

The Finite element model is shown in Fig.W

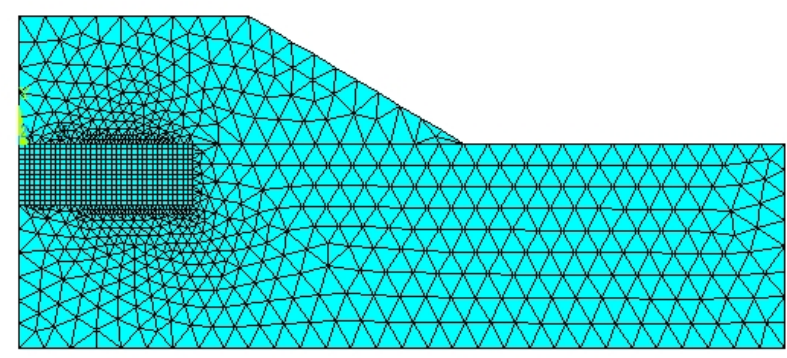

Figure 1: Finite element mode.

\subsection{Parameter Setting}

Steps for MD transient thermal simulation include Create the model,Applied load and solution, Reprocessing. 
For the model of MD, the air temperature on the surface of soil is only the input factor, the water temperature in MD is the output factor.

When the applied load, the air temperature on the surface of soil is fitted as a function:

$$
T=P_{0}+P_{1} \times \sin \frac{(2 \times \pi) \times\left(T I M E-P_{3}\right)}{P_{2}}
$$

Where $P_{0}$ is the average annual air temperature on the surface of soil. According to the empirical formula, $P_{0}$ should be 2 more than the real value; $P_{1}$ is the amplitude for air temperature on the surface of soil; $P_{2}$ is the period for air temperature on the surface of soil; $P_{3}$ is the phase deviation for air temperature on the surface of soil; TIME is time (day); $T$ is the air temperature on the surface of soil with corresponding time.

The calculation results are obtained by finite element simulation.

\section{The data analysis}

\subsection{Simulation data and measured data analysis for MD at Yangbajing Tibet}

Two MD prototypes were built respectively at Yangbajing Tibet in 2012 and 2014. In order to verify the correctness of MD model, a simulation has been made using the basic parameters from the Yangbajing Tibet.

Basic parameters were shown in Table.W:

Table 1: Thermodynamic calculation paramete.

\begin{tabular}{|l|c|c|c|}
\hline Material & $\begin{array}{c}\text { Density } \\
\left(\mathrm{Kg} / \mathrm{m}^{3}\right)\end{array}$ & $\begin{array}{c}\text { Thermal conductivity } \\
\left(\mathrm{W} / \mathrm{m}^{2} \cdot{ }^{\circ} \mathrm{C}\right)\end{array}$ & $\begin{array}{c}\text { Specific heat capacity } \\
(\mathrm{J} / \mathrm{Kg} \cdot \mathrm{C})\end{array}$ \\
\hline Water & 996 & 0.599 & 4185 \\
\hline Soil & 1450 & 0.4 & 840 \\
\hline Air & 1.293 & 0.024 & 1000 \\
\hline Reinforced Concrete & 3000 & 0.38 & 1880 \\
\hline
\end{tabular}

The average annual air temperature of Yangbajing Tibet is shown in Fig.D:

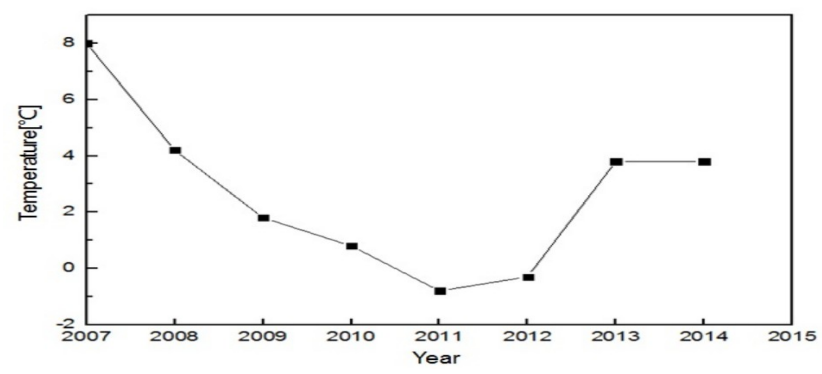

Figure 2: The annual average air temperature of Yangbajing Tibet.

The air temperature on the surface of soil at Yangbajing Tibet is shown in Fig.[3]: 


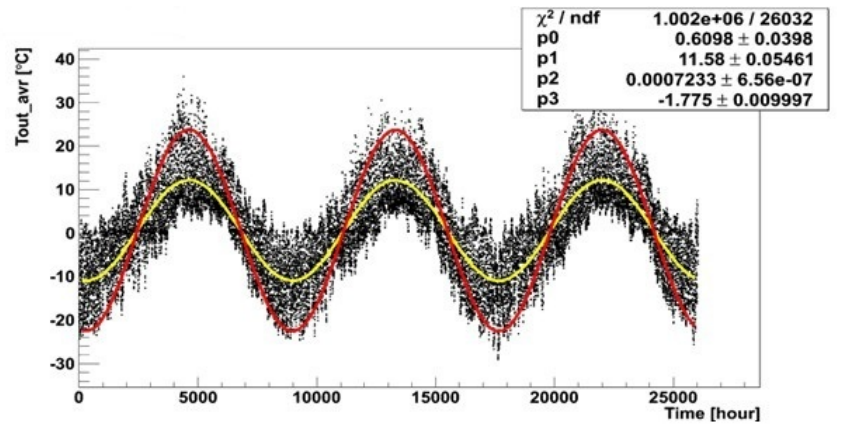

Figure 3: The air temperature on the surface of soil at Yangbajing Tibet.

Where the yellow line is the average air temperature fitting function; the red line is the limit air temperature which is fitted as the function 4.1.

$$
T=2.7+15.7 \times \sin \frac{(2 \times \pi) \times(T I M E-273)}{365}
$$

The simulation data of water temperature in MD is compared with the measured data, and the results are shown in Fig.⿴囗十 - Fig. [D.

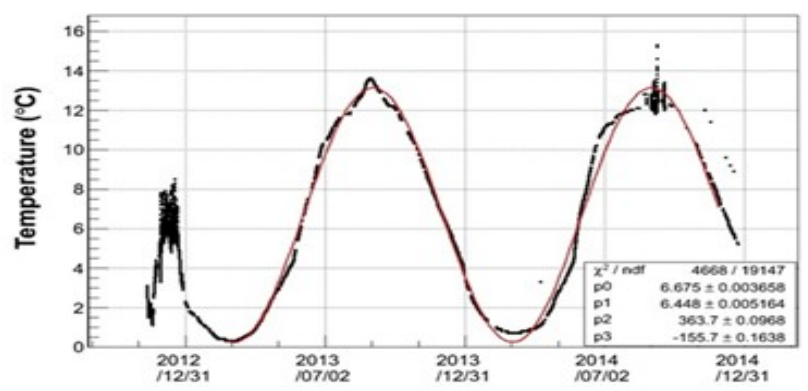

Figure 4: Measured data of water temperature in $\operatorname{MD}(2012)$.

Fig. 田 is the measured data of water temperature in MD, which was built in 2012. The MD tank was placed on the ground. Fitting the measured data, $P_{0}=6.7 ; P_{1}=6.4 ; P_{2}=364 ; P_{3}=156$.

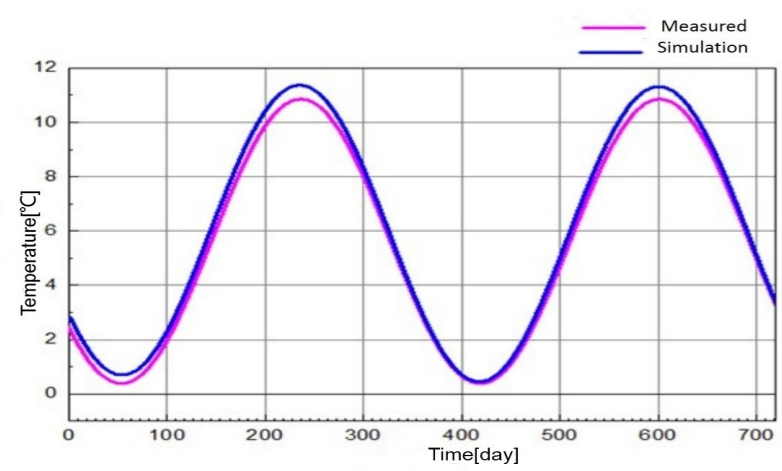

Figure 5: Water temperature comparison between simulated and measured(2012). 
Fig.[1 is water temperature in MD comparison between simulated and measured. The blue line is simulation data, the red line is measured data. The difference between the measured and simulation data is less than $1^{\circ} \mathrm{C}$.

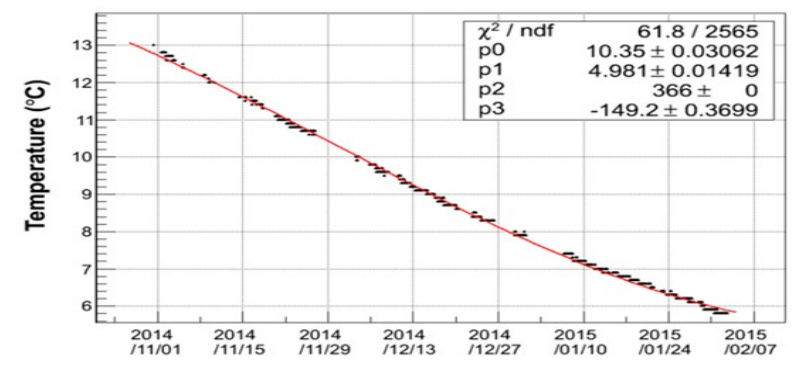

Figure 6: Measured data of water temperature in MD(2014).

Fig.6 is the measured data of water temperature in MD, which was built in 2014. The MD tank was placed on the ground. Fitting the measured data, $P_{0}=10.35 ; P_{1}=4.98 ; P_{2}=366 ; P_{3}=150$.

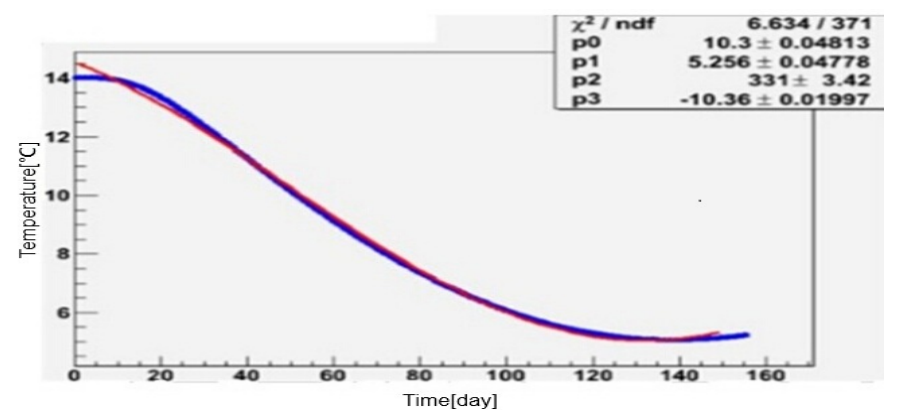

Figure 7: Water temperature comparison between simulated and measured(2014).

Fig. $\square$ is water temperature in MD comparison between simulated and measured. The blue line is simulation data, the red line is measured data. The difference between the measured and simulation data is less than $1^{\circ} \mathrm{C}$.

One MD tank was placed on the ground, another MD tank was buried deep underground. For Two MD prototypes, as shown in Table.l, measured data is approximate to respective simulation data, it proves that the MD model is correct.

Table 2: Water temperature comparison between simulated and measured.

\begin{tabular}{|c|c|c|c|c|c|c|}
\hline Similarity & Peak & Valley & Mean & Slope & Phase & Phase \\
\hline Data & $82 \%$ & $85 \%$ & $85 \%$ & $92 \%$ & 100 Day & 90 Day \\
\hline
\end{tabular}

\subsection{Simulation data and measured data analysis for MD at DaoCheng SiChuan}

Muon Detectors will be built at Daocheng Sichuan province. In order to study MDs antifreeze design at DaoCheng, a simulation which is the same model as Yangbajing Tibet has been made using the new site basic parameters. 


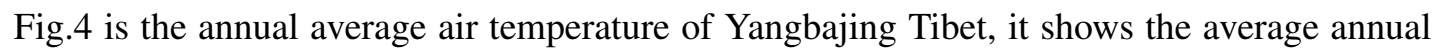
temperature fluctuate $5^{\circ} \mathrm{C}$ during eight years. To preventation of extreme cases, the case of annual average $5^{\circ} \mathrm{C}$ drop is considered in the simulation.

Because of terrain restrictions, not all MDs is buried in the ground. According to the location of the MD in the soil, there are three types. Base on the bottom of the MD tank, Marsh type, the MD can be placed on the ground; Shallow type, the MD should be buried at $1.2 \mathrm{~m}$ underground; Deep type, the MD should be buried at $4.2 \mathrm{~m}$ underground.

Fig. 8 shows the simulation result.

\begin{tabular}{|c|c|c|c|}
\hline Type & Antifreeze layer & $\begin{array}{c}\text { Input Factor } \\
\text { (Air temperature) }\end{array}$ & $\begin{array}{c}\text { Output Factor } \\
\text { (Min water temperature) }\end{array}$ \\
\hline \multirow{2}{*}{ Marsh } & \multirow{2}{*}{$15 \mathrm{~cm}$} & Normal & $2.23^{\circ} \mathrm{C}$ \\
\hline & & Extreme & $0.55^{\circ} \mathrm{C}$ \\
\hline \multirow{2}{*}{ Shallow } & \multirow{2}{*}{$10 \mathrm{~cm}$} & Normal & $2.58^{\circ} \mathrm{C}$ \\
\hline & & Extreme & $0.73^{\circ} \mathrm{C}$ \\
\hline \multirow{2}{*}{ Deep } & \multirow{2}{*}{$10 \mathrm{~cm}$} & Normal & $2.12^{\circ} \mathrm{C}$ \\
\hline & & Extreme & $1.06^{\circ} \mathrm{C}$ \\
\hline
\end{tabular}

Figure 8: Simulation resul for DaoCheng.

Consider the models error, a conclusion can be obtained:

Where Marsh type, $15 \mathrm{~cm}$ antifreeze layer should be adopted, it ensures the lowest water temperature of $\mathrm{MD}$ is $2^{\circ} \mathrm{C}-4^{\circ} \mathrm{C}$, extreme temperature above $0^{\circ} \mathrm{C}$; Shallow type, $10 \mathrm{~cm}$ antifreeze layer should be adopted, it ensures the lowest water temperature of MD is $2^{\circ} \mathrm{C}-4^{\circ} \mathrm{C}$, extreme temperature above $0^{\circ} \mathrm{C}$; Deep type, $10 \mathrm{~cm}$ antifreeze layer should be adopted, it ensures the lowest water temperature of $\mathrm{MD}$ is $2^{\circ} \mathrm{C}-4^{\circ} \mathrm{C}$, extreme temperature above $0^{\circ} \mathrm{C}$.

To verify the simulation results, three type of MDs were built at Daocheng Sichuan province in 2015. Water temperature in the MD center and water temperature in the MD boundary and the soil temperature outside the MD were measured, every position have three temperature sensors. Fig.\$ is the measured data of water temperature in marsh type MD. The blue lines are the water temperature in the MD center, the red lines are the water temperature of the water in the MD boundary, the black lines are the soil temperature outside the MD. Fig.Q 9 shows that measured data is approximate to respective simulated data, it proves that the simulation results are correct, antifreeze design is feasible.

In addition, through the measured data, it can be found that the water temperature changes more quickly between $3.5^{\circ} \mathrm{C}$ and $4^{\circ} \mathrm{C}$, the reason is that water density is the maximum at $4^{\circ} \mathrm{C}$, water convection movement up and down at this temperature, consequently accelerate heat exchange.

\section{Summary and Conclusions}

Studying the water temperature of two MD prototypes at Yangbajing Tibet, measured data is approximate to respective simulation data, it proves that the MD model is correct.

Studying the water temperature of three MD prototypes at Daocheng Sichuan, measured data is approximate to respective simulation data, it proves that antifreeze design is feasible. 


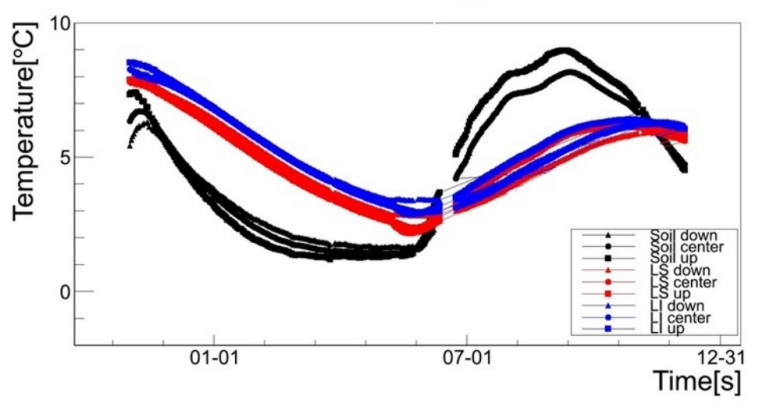

Figure 9: Measured data of water temperature in MD (Marsh Type).

Consider the Antifreeze performance and price, Polyurethane is used as an anti-freeze material for MD array. Marsh type, $15 \mathrm{~cm}$ antifreeze layer should be adopted; Shallow type, $10 \mathrm{~cm}$ antifreeze layer should be adopted; Deep type, $10 \mathrm{~cm}$ antifreeze layer should be adopted.

\section{Acknowledgements}

This work is supported by National Natural Science Foundation (NSFC) of China under contacts No.11635011.

\section{References}

[1] Z. Cao et al., LHAASO Collaboration, 31st ICRC, Lodz, (2009).

[2] Z. Cao., LHAASO Coll., CPC 34(2) (2010) 249-252.

[3] Z. Cao., LHAASO Coll., Frascati Physics Series Vol. 58(2014).

[4] X. Zuo, LHAASO Coll., Nuclear Instruments and Methods in Physics Research Section A 789 (2015) 143-149.

[5] Xinhua. Ma et al., LHAASO Coll., Astroparticle Physics 54 (2014) 86-92.

[6] J. W. Cronin, Nucl. Phys. B(Proc. Suppl), 2005, 138, 465.

[7] M. A. K. Glasmacher, Astropart. Phys. 1999, 12, 1.

[8] J. Zabierowski et al., KASCADE-Grande Coll., Proc. of 30th ICRC, Merida (2007), vol. 4: 111-114.

[9] W. D. Apel. et al., Astropart. Phys., 2011(34): 476-485.

[10] J.C. Arteaga et al., Proc. 33st ICRC, RIO DE JANEIRO, 2013, astroph.HE, arXiv:1308.3202v1.

[11] V. Souza et al., Proc. of the 32nd ICRC, Beijing (2011), ID 0953. 